\title{
A Multi-Frame Optical Flow Spot Tracker
}

Jizhou Li, Christopher Gilliam and Thierry Blu

Department of Electronic Engineering, The Chinese University of Hong Kong

\section{Summary}

Accurate and robust spot tracking is a necessary tool for quantitative motion analysis in fluorescence microscopy images. In this work, we exploits the underlying stationary motion in biological systems, e.g. the movement of crowds, bacteria swarming and cyclosis in plant cells, and then propose a multi-frame optical flow based tracker. We obtain the stationary motion by adapting a recent optical flow algorithm that relates one image to another locally using an all-pass filter. We perform this operation over all the image frames simultaneously and estimate a single, stationary optical flow. We compare the proposed tracker with two existing techniques and show that our Weproach is more robust to high noise and varying structure. In addition, we also show initial experiments on real microscopy images.

\section{Spot Tracking}

Problem: To detect and track a set of spots over an image sequence comprising $N$ frames

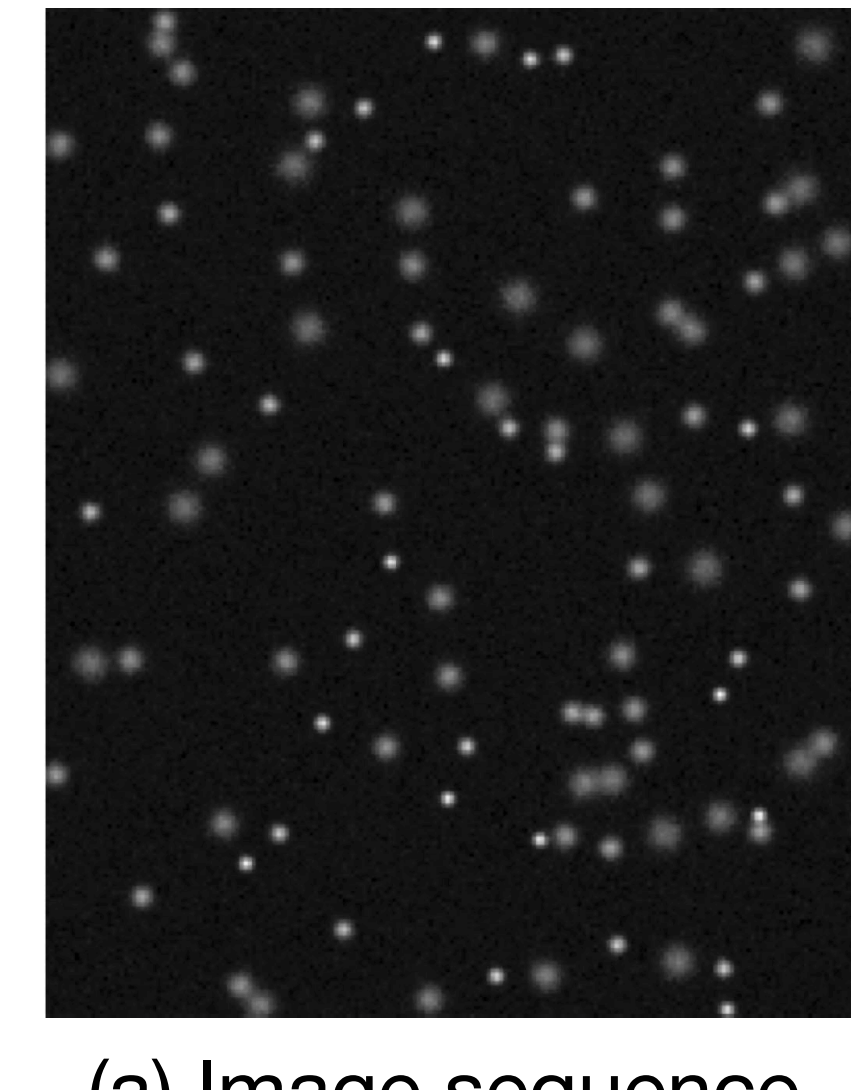

(a) Image sequence

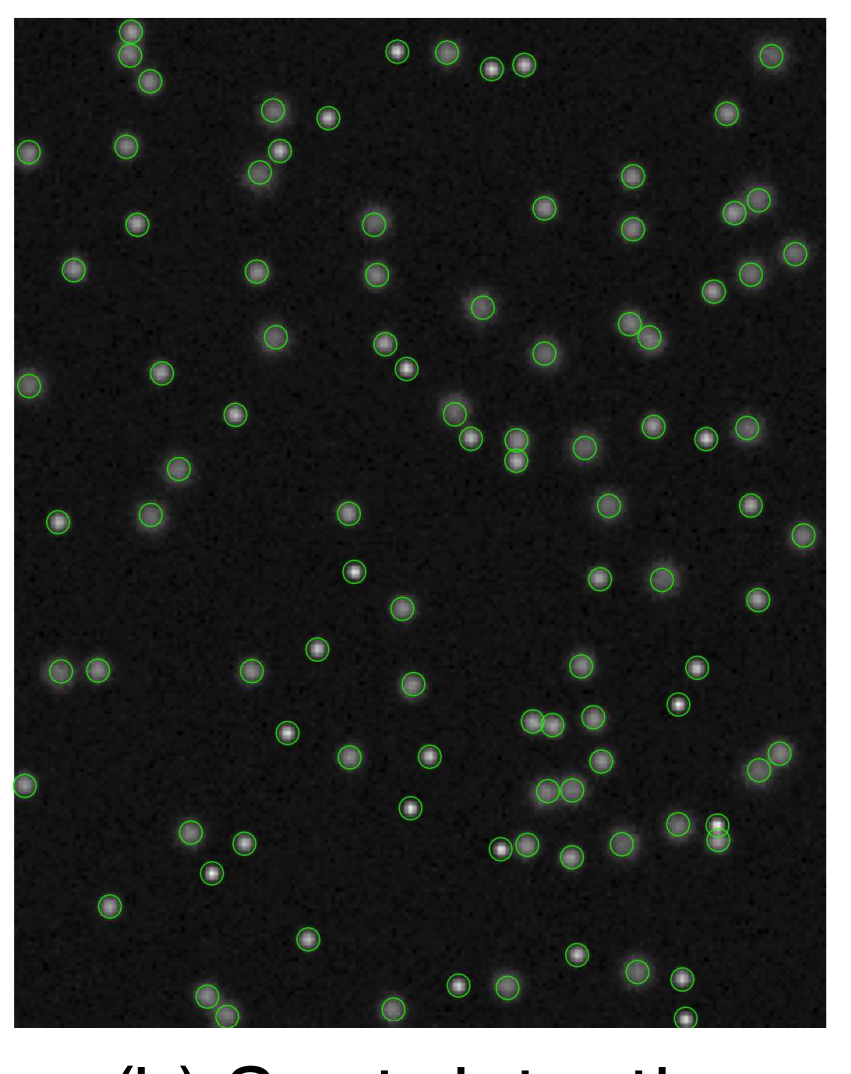

(b) Spot detection

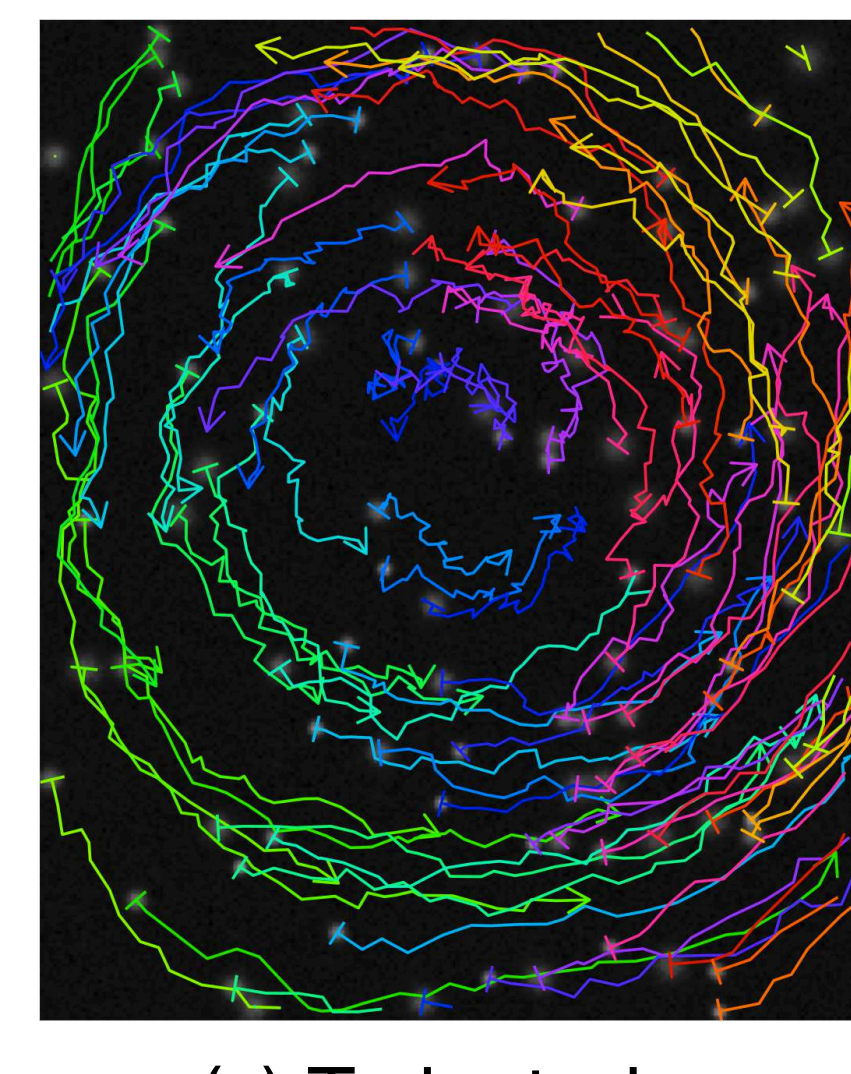

(c) Trajectories

\section{Our Approach}

(a) Stationary Motion

Assumption: there exists a motion pattern that underpins the dynamic within the system being imaged, called the stationary motion field.

This underlying motion is the main part of the motion between two consecutive images that is consistent across all the images in the sequence.

This type of motion has been observed in many organisms:

- Crowds of people

- Flocks of birds

- Bactería

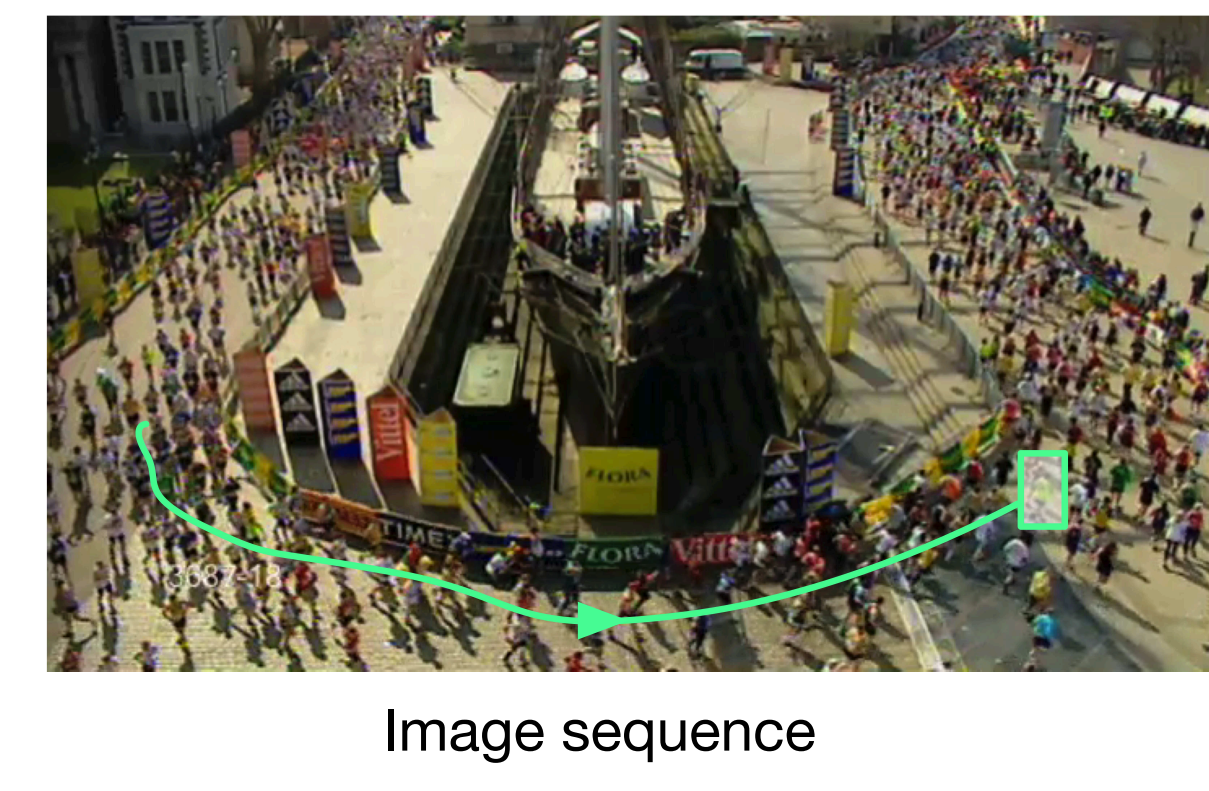

(b) Stationary Local All-Pass (SLAP) Tracker

Step 1: Estimation of an underlying stationary motion field over all image frames simultaneously using an adapted version of the local all-pass algorithm [1]

Step 2: Simple spot detection

Step 3: Trajectory estimation based on the estimated stationary motion filed.

$\leftrightarrow$ Use the stationary motion to guide the spot tracking

\section{Stationary Motion Estimation}

1. Motion estimation using Local All-Pass (LAP) filters

The LAP algorithm proposed in [1]

- Constant motion $\Longrightarrow$ All-pass filtering

- Relate local windows in two images using this filter

- Extract the motion vectors from the local all-pass filters

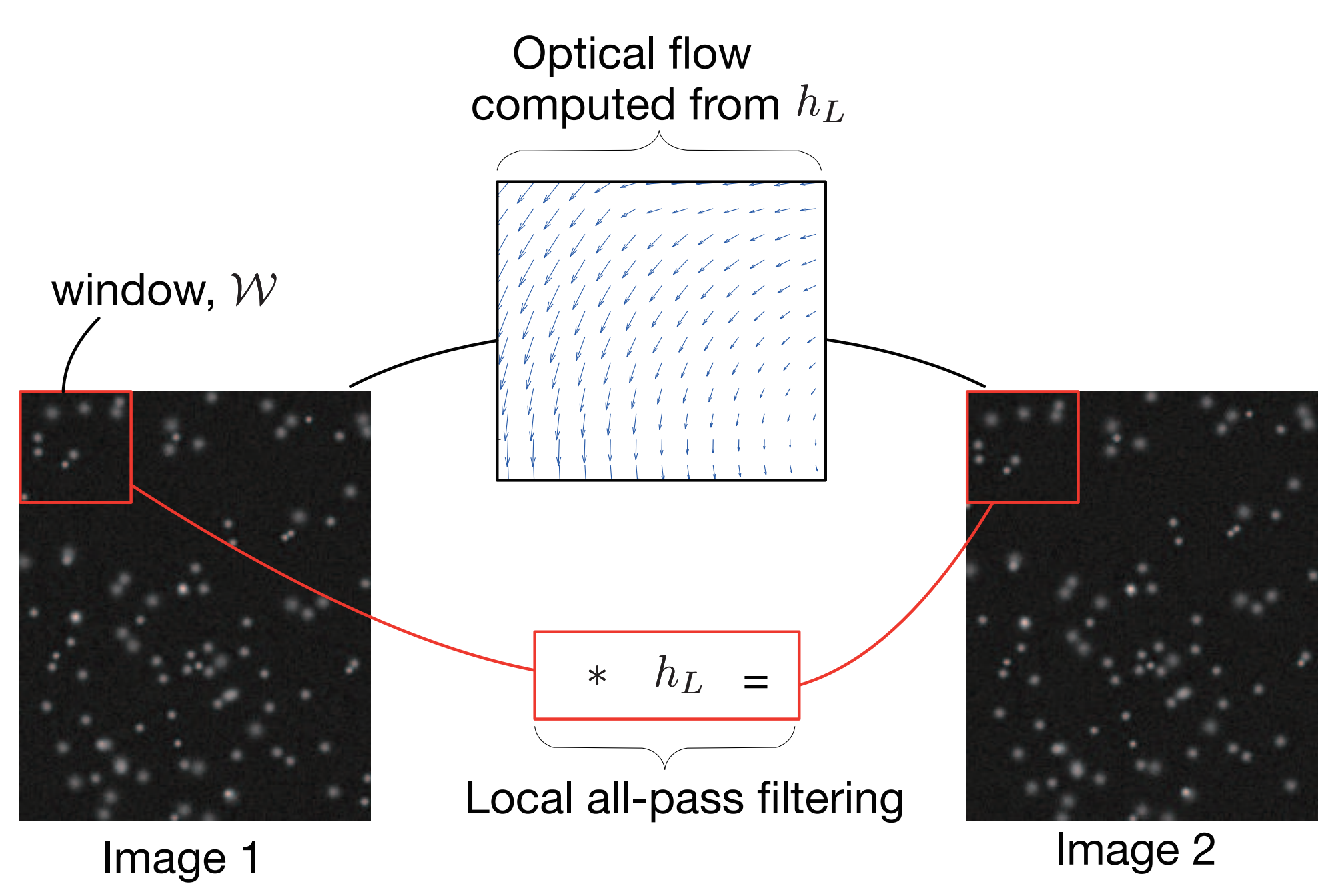

2. Adaption to stationary motion

We estimate one single motion field from the whole image sequence simultaneously.

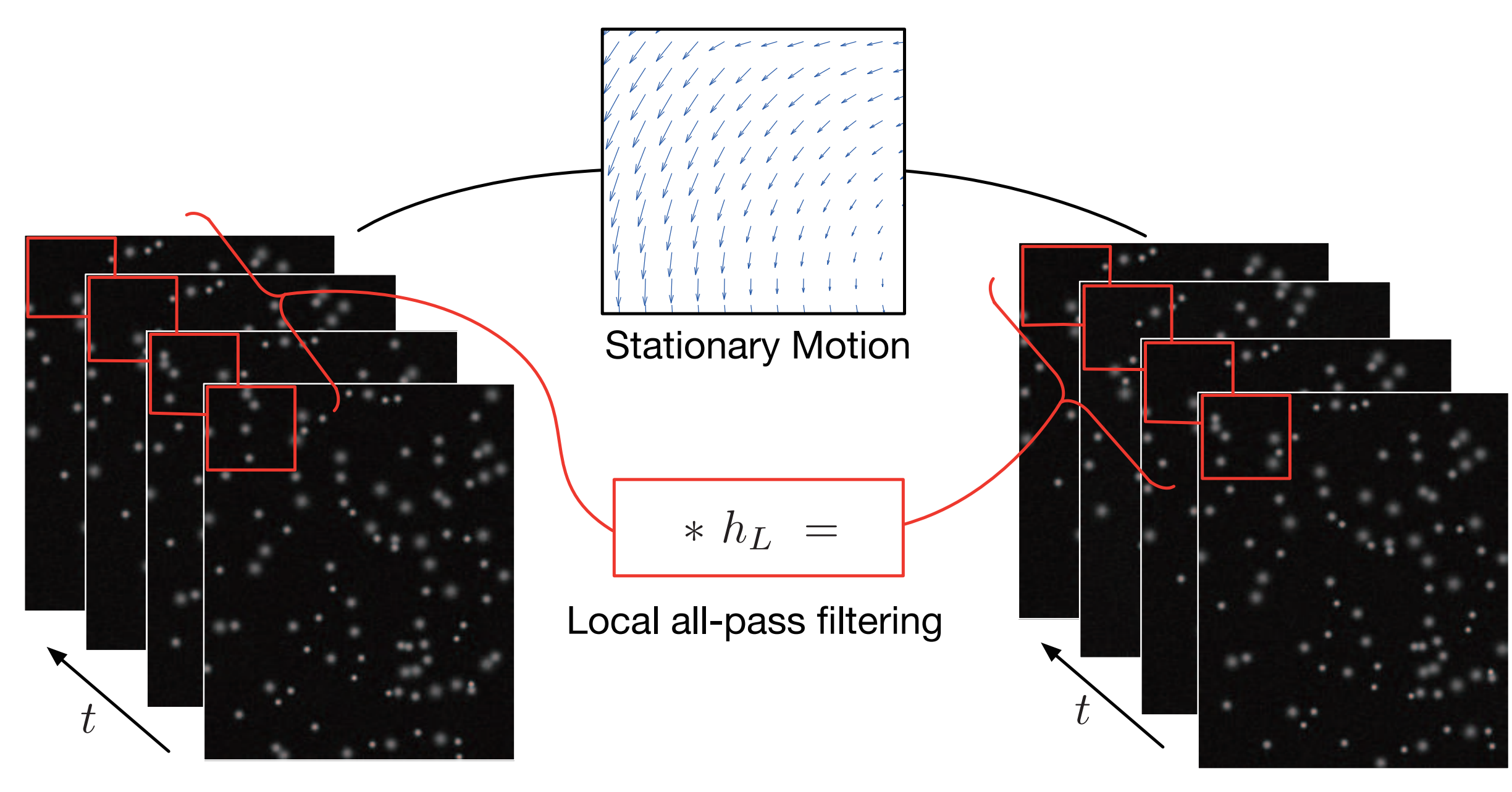

$\rightarrow$ Extract the stationary motion field $\bar{u}$ from these filters.

\section{Detection and Trajectory Estimation}

Spots Detection: The spot locations are estimated by finding the local maxima of the correlations with a pre-defined Gaussian mask.

Trajectory Estimation: The trajectory is built based on associating the spot in the next frame that is closest to the predicted position.

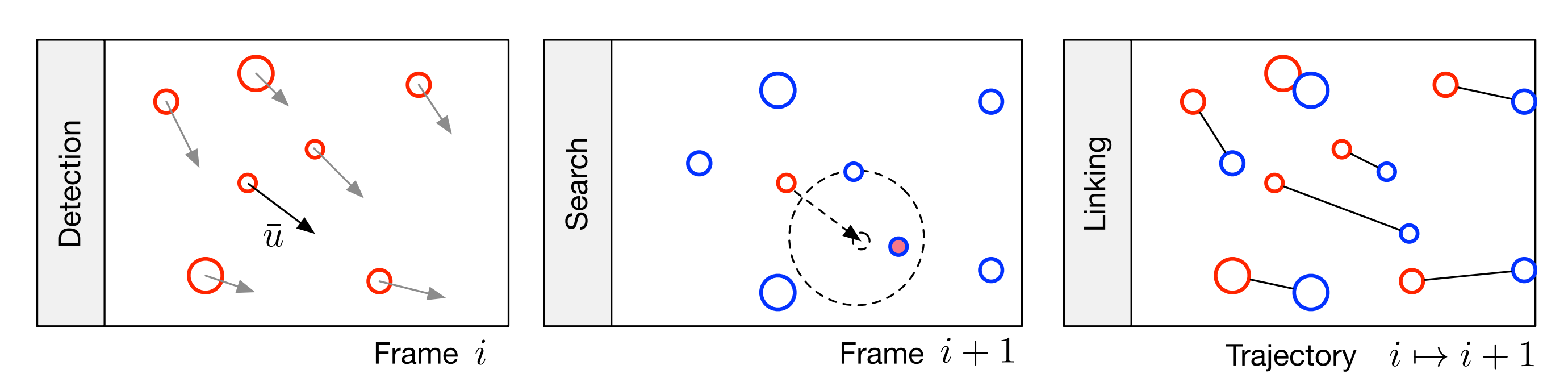

\section{Experiment and Results}

\section{Performance Evaluation:}

We evaluate a tracker based upon the percentage of correctly identified trajectories

\section{Experiment and Results}

Synthetic Images: The synthetic image sequence consists of two elements: the spots we wish to track and the underlying stationary motion that controls their movement.
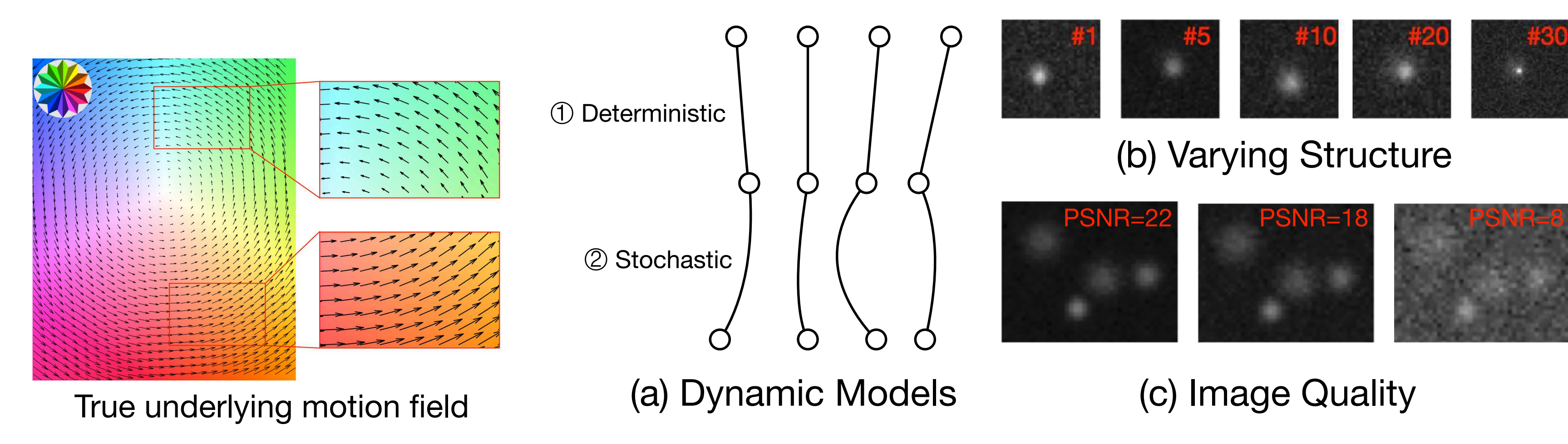

Results:

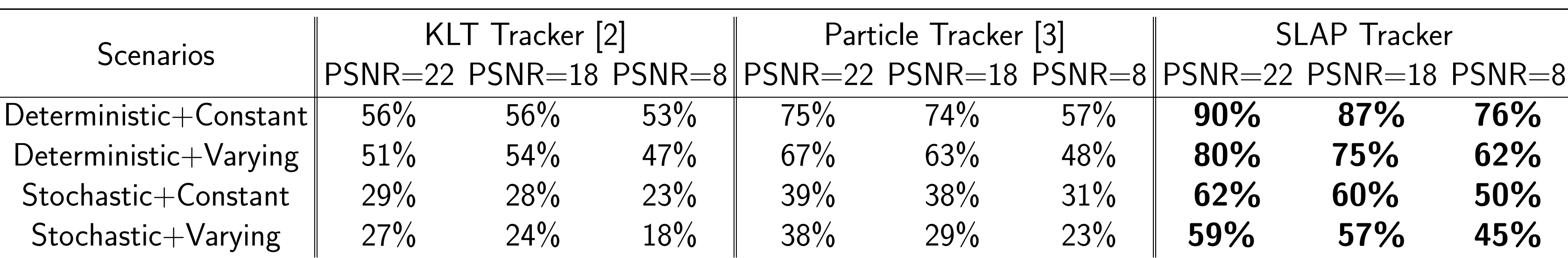

$\leftrightarrow$ The simple detection and linking strategies have low computational cost. $\rightarrow$ The stationary motion field is only calculated once across all the images using

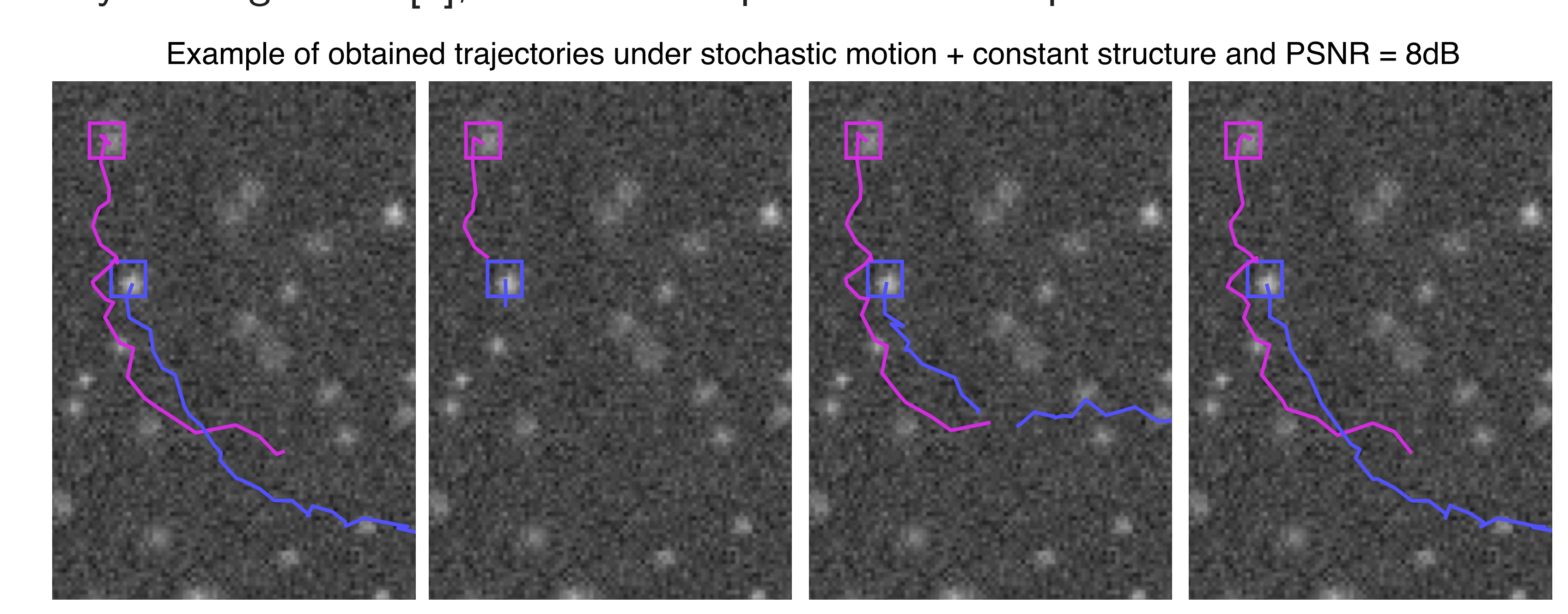

Ground Truth

KLT Tracker

Particle Tracker

SLAP Tracker

Real Images:

- The sequence depicts cytoplasmic streaming in Drosophila oocytes (fruit fly eggs) [4].

- The SLAP Tracker successfully followed 25 spots (correctness = 83.3\%) during 30 consequent frames
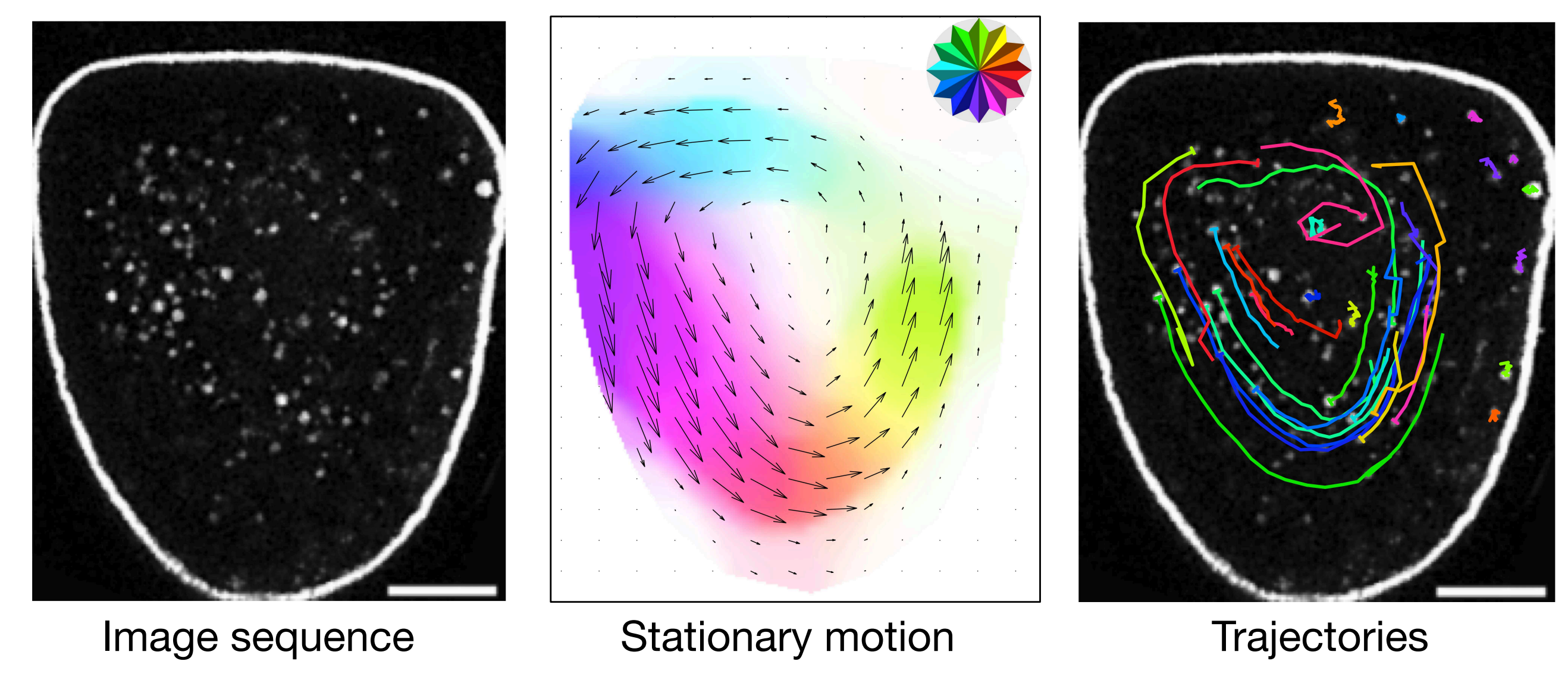

\section{References}

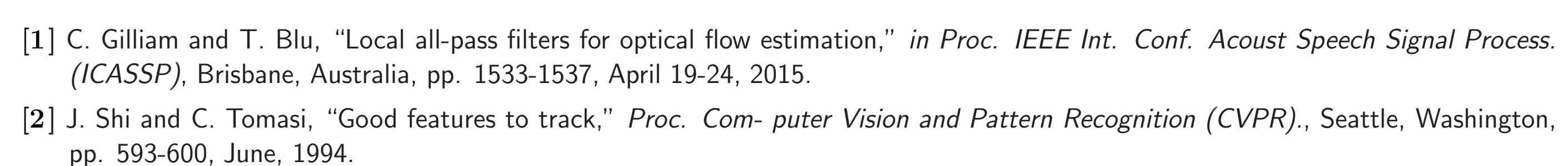

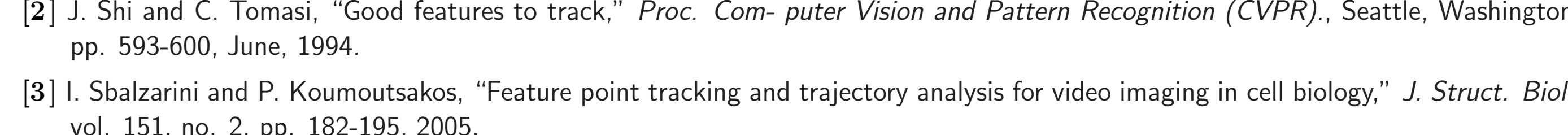

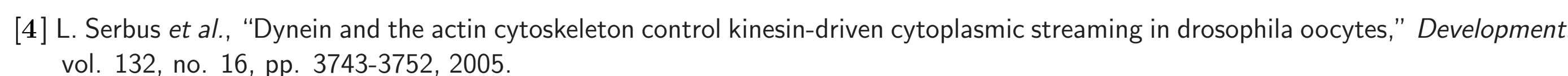

